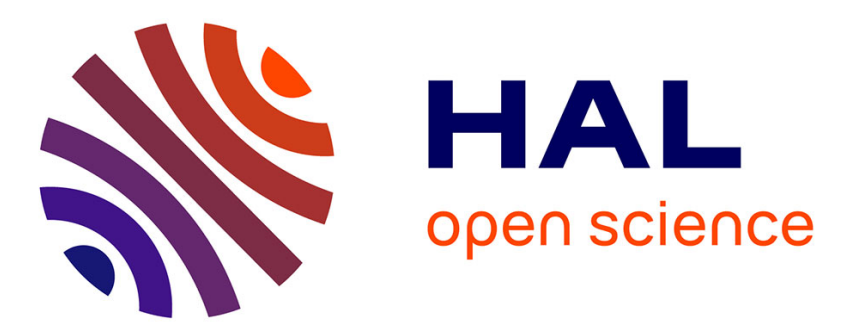

\title{
The Laplace equation in 3-D domains with cracks: Dual shadows with log terms and extraction of corresponding edge flux intensity functions
}

\author{
Samuel Shannon, Victor Péron, Zohar Yosibash
}

\section{- To cite this version:}

Samuel Shannon, Victor Péron, Zohar Yosibash. The Laplace equation in 3-D domains with cracks: Dual shadows with log terms and extraction of corresponding edge flux intensity functions. Mathematical Methods in the Applied Sciences, 2016, 10.1002/mma.3562 . hal-01111593

\author{
HAL Id: hal-01111593 \\ https://hal.inria.fr/hal-01111593
}

Submitted on 6 Jul 2015

HAL is a multi-disciplinary open access archive for the deposit and dissemination of scientific research documents, whether they are published or not. The documents may come from teaching and research institutions in France or abroad, or from public or private research centers.
L'archive ouverte pluridisciplinaire HAL, est destinée au dépôt et à la diffusion de documents scientifiques de niveau recherche, publiés ou non, émanant des établissements d'enseignement et de recherche français ou étrangers, des laboratoires publics ou privés. 


\title{
The Laplace equation in 3-D domains with cracks: Dual shadows with log terms and extraction of corresponding edge flux intensity functions ${ }^{\dagger}$
}

\author{
Samuel SHANNON *, Victor PERON \& and Zohar YOSIBASH *
}

The singular solution of the Laplace equation with a straight-crack is represented by a series of eigenpairs, shadows and their associated edge flux intensity functions (EFIFs). We address the computation of the EFIFs associated with the integer eigenvalues by the quasi dual function method (QDFM).The QDFM is based on the dual eigenpairs and shadows, and we show that the dual shadows associated with the integer eigenvalues contain logarithmic terms. These are then used with the QDFM to extract EFIFs from p-version finite element solutions. Numerical examples are provided. Copyright (C) 0000 John Wiley \& Sons, Ltd.

Keywords: Logarithmic singularities; dual eigenvalues; edge flux/stress intensity functions; quasi-dual function method; 3-D singularities

\section{Dedicated to the 65th birthday of prof. Martin Costabel.}

\section{Introduction and notation}

Solutions of the Laplace equation in the vicinity of singular points over two dimensional domains have been investigated for over half a century. These are described by an infinite series of eigenpairs and their coefficients named flux intensity factors (FIFs). The eigenpairs depend on the boundary conditions on the crack faces (see [14]). For example for crack with homogeneous Dirichlet boundary conditions (BCs) the eigenvalues $\alpha_{k}$ are: $\alpha_{k}=\cdots-2,-3 / 2,-1,-1 / 2,1 / 2,1,3 / 2,2 \cdots$. For homogeneous Neumann boundary conditions same eigenvalues are obtained in addition of 0 . The eigenpairs associated with the negative eigenvalues are called dual eigenpairs. They are not a part of the solution because they are not in the Sobolev space $H^{1}$ (do not belong to the energy space). The integer eigenpairs and their corresponding coefficients, especially associated with $\alpha=1$, are important from the engineering point of view, as they may affect the path stability of slightly curved or kinked cracks $[4,8]$, and play an important role in determining the size and shape of a crack tip plastic zone in the theory of elasticity.

In three dimensional domains such as polyhedra the Laplace equation, in cylindrical coordinates $(\rho, \varphi, z)$ located on the straight singular edge, is (see Figure 1):

$$
\Delta_{(\rho, \varphi, z)} \tau=\left\{\partial_{\rho \rho}+\frac{1}{\rho} \partial_{\rho}+\frac{1}{\rho^{2}} \partial_{\varphi \varphi}+\partial_{z z}\right\} \tau=0
$$

For a straight singular edge, the solution of the Laplace equation is represented by two infinite series, the 2-D eigenpairs series and another series composed of shadow functions and the derivatives of the edge flux intensity functions (EFIFs) which are functions along the edge (see [5, 13]). Explicit representation of the solution in terms of eigenpairs, shadows and EFIFs is (see

\footnotetext{
* Pearlstone Center for Aeronautical Eng. Studies, Dept. of Mechanical Engineering, Ben-Gurion University of the Negev, Beer-Sheva, 84105, Israel, Tel.:+972-8-6477103, Fax:+972-8-6477101 E-mail: zohary@bgu.ac.il

\& INRIA-Bordeaux Sud Ouest, LMA - UMR CNRS 5142, Université de Pau et des Pays de l'Adour, 64013 Pau cedex, France, Tel.: + 33-5-40175209,

E-mail: victor.peron@univ-pau.fr
} 


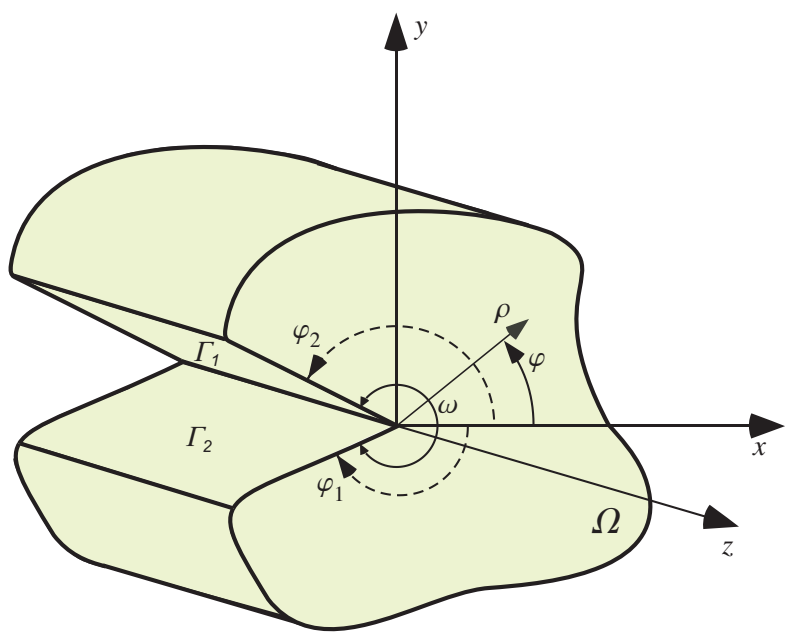

Figure 1. A 3-D domain containing a straight singular edge at $(x, y)=(0,0)$

$[7,3,12]):$

$$
\tau(\rho, \varphi, z)=\sum_{k} \sum_{\ell=0,2,4,6 \cdots}^{\infty} \partial_{z}^{\ell} A_{k}(z) \rho^{\alpha_{k}+\ell} \phi_{k, \ell}(\varphi)
$$

where $\alpha_{k}$, are the 2-D eigenvalues (for a crack with homogeneous Dirichlet BCs the eigenvalue are $\alpha_{k}=k / 2, k=1,2,3, \cdots$ and for a crack with homogeneous Neumann BCs $\left.\alpha_{k}=k / 2, k=0,1,2, \cdots\right), \phi_{k, \ell}(\varphi)$ are the 2-D eigenfunctions and their shadows and $A_{k}(z)$ are the edge flux intensity functions (EFIFs).

The EFIFs may be computed using the dual eigenpairs by an extraction method named the quasi-dual function method (QDFM) (See [3, 12]). The QDFM has been successfully applied for the extraction of EFIFs associated with the non-integer eigenvalues from finite element (FE) solutions, but it does not apply to EFIFs associated with the integer eigenvalues (see [3]). Other methods for extracting EFIFs are available (see e.g. $[1,11]$ ), however these methods do not apply to integer eigenvalues either.

We first demonstrate that the dual shadows associated with integer eigenvalues for the Laplace operator over a 3-D domain with a straight crack involve logarithmic terms. This is an interesting observation because it was proven in [2] that the asymptotic solution in the vicinity of cracks under homogeneous Dirichlet or Neumann BCs do not contain logarithmic terms. Using the dual and shadow functions with the logarithmic terms we extend the QDFM for the extraction of the EFIFs associated with the integer eigenvalues. We implement the presented methods and provide numerical examples using the $\mathrm{p}$-version of the FE method.

\section{The asymptotic solution in the vicinity of a straight 3-D crack front}

The solution to (1) in the vicinity of a straight singular edge (crack as a particular example) is given by (2) (see [3]), with homogeneous Dirichlet or Neumann BCs on the crack faces, that imply:

$$
\begin{array}{r}
\phi_{k, \ell}(\varphi= \pm \pi)=0, \quad \text { Homogeneous Dirichlet BCs } \\
\phi_{k, \ell}^{\prime}(\varphi= \pm \pi)=0, \quad \text { Homogeneous Neumann BCs }
\end{array}
$$

Substituting (2) in (1) one obtains:

$$
\begin{aligned}
& \Delta \tau=\rho^{\alpha_{k}-2}\left[A_{k}(z)\left\{\alpha_{k}^{2} \phi_{k, 0}(\varphi)+\phi_{0,0}^{\prime \prime}(\varphi)\right\}\right. \\
&+A_{k}^{\prime \prime}(z) \rho^{2}\left\{\left(\alpha_{k}+2\right)^{2} \phi_{k, 2}(\varphi)+\phi_{k, 2}^{\prime \prime}(\varphi)+\phi_{k, 0}^{\prime \prime}(\varphi)\right\} \\
&+A_{k}^{(4)}(z) \rho^{4}\left\{\left(\alpha_{k}+4\right)^{2} \phi_{k, 4}(\varphi)+\phi_{k, 4}^{\prime \prime}(\varphi)+\phi_{k, 2}^{\prime \prime}(\varphi)\right\} \\
&+A_{k}^{(6)}(z) \rho^{6}\left\{\left(\alpha_{k}+6\right)^{2} \phi_{k, 6}(\varphi)+\phi_{k, 6}^{\prime \prime}(\varphi)+\phi_{k, 4}^{\prime \prime}(\varphi)\right\} \\
&+\cdots]=0
\end{aligned}
$$

Since (5) is equal to zero for any $\rho^{i}$, the following recursive set of ODEs is obtained that together with the boundary conditions determine the eigenvalues, eigenfunctions and their shadows:

$$
\begin{array}{cl}
\alpha_{k}^{2} \phi_{k, 0}(\varphi)+\phi_{k, 0}^{\prime \prime}(\varphi)=0, & \ell=0 \\
\left(\alpha_{k}+\ell\right)^{2} \phi_{k, \ell}(\varphi)+\phi_{k, \ell}^{\prime \prime}(\varphi)+\phi_{k, \ell-2}^{\prime \prime}(\varphi)=0, & \ell \geq 2, \ell \in 2 \mathbb{N}
\end{array}
$$


Equation (6) with the BC (3) or (4) is identical to the one obtained for the 2-D Laplace problem with the 2-D eigenvalues $\alpha_{k}$ and the corresponding eigenfunctions $\phi_{k, 0}$. For a crack with homogeneous Neumann BCs the eigenvalues are:

$$
\alpha_{k}=\cdots-3,-\frac{5}{2},-2,-\frac{3}{2},-1,-\frac{1}{2}, 0, \frac{1}{2}, 1, \frac{3}{2}, 2, \frac{5}{2}, 3 \cdots
$$

whereas for the homogeneous Dirichlet BCs same eigenvalues are obtained excluding $\alpha_{0}=0$. We denote by $\tau$ the solution of the Laplace equation constructed by the positive eigenpairs and their shadows $(2) . A_{k}(z)$ are the edge flux intensity functions, and $\phi_{k, \ell}$ with $\ell \neq 0$ are the shadow functions. The negative eigenvalues and the corresponding eigenfunctions are not a part of the solution because they are not in the Sobolev space $H^{1}$, and these form the "dual solution". We define by $K(\rho, \varphi, z)$ the "dual solution":

$$
K(\rho, \varphi, z)=\sum_{k} \sum_{\ell=0,2,4,6 \ldots}^{\infty} \partial_{z}^{\ell} B_{k}(z) \rho^{-\alpha_{k}+\ell} \psi_{k, \ell}(\varphi)
$$

The dual eigenfunctions $\psi_{k, 0}$ and their shadow functions $\psi_{k, \ell}$ are obtained by the recursive relationship (here $\alpha_{k}$ are negative):

$$
\begin{array}{cl}
\alpha_{k}^{2} \psi_{0}(\varphi)+\psi_{0}^{\prime \prime}(\varphi)=0, & \ell=0 \\
\left(\alpha_{k}+\ell\right)^{2} \psi_{k, \ell}(\varphi)+\psi_{k, \ell}^{\prime \prime}(\varphi)+\psi_{k, \ell-2}^{\prime \prime}(\varphi)=0, & \ell \geq 2, \ell \in 2 \mathbb{N}
\end{array}
$$

Remark 1 We show in the sequel that for the integer dual eigenvalues (i.e. $\alpha_{k}=0,-1,-2, \ldots$ ) the solution of equations (9)-(10) without logarithmic terms cannot satisfy the BCs and therefore logarithmic terms will be present in (8).

\subsection{Computation of eigenfunctions and shadows for a crack with homogeneous Dirichlet BCs}

Substituting $\alpha_{1}=\frac{1}{2}$ in (6) with homogeneous Dirichlet BCs one obtains:

$$
\phi_{1,0}(\varphi)=\cos \frac{\varphi}{2}
$$

To obtain $\phi_{1,2}(\varphi)$ we substitute (11), $\alpha_{1}=\frac{1}{2}$ and $\ell=2$ in (7):

$$
\left(\frac{1}{2}+2\right)^{2} \phi_{1,2}(\varphi)+\phi_{1,2} "(\varphi)=-\cos \frac{\varphi}{2}
$$

The solution of (12) with homogeneous Dirichlet BCs is:

$$
\phi_{0,2}(\varphi)=-\frac{1}{6} \cos \frac{\varphi}{2}
$$

Remark 2 Equation (13) is the particular solution of (12). As it satisfies (12) and the homogeneous Dirichlet BCs (3), for simplicity, we choose the coefficient of the homogeneous solution of (12) to be zero.

Equations (6)-(7) can be solved for $\alpha_{k}=1 / 2,1,3 / 2,2$, obtaining $\phi_{1, \ell}, \phi_{2, \ell}, \phi_{3, \ell}, \phi_{4, \ell}$. These yield the following series solution for a straight crack with homogeneous Dirichlet BCs:

$$
\begin{aligned}
\tau & =\rho^{1 / 2}\left\{A_{1}(z) \cos \frac{\varphi}{2}-A_{1}^{\prime \prime}(z) \rho^{2} \frac{1}{6} \cos \frac{\varphi}{2}+A_{1}^{(4)}(z) \rho^{4} \frac{1}{120} \cos \frac{\varphi}{2}-A_{1}^{(6)}(z) \rho^{6} \frac{1}{5040} \cos \frac{\varphi}{2}+\cdots\right\} \\
& +\rho\left\{A_{2}(z) \sin \varphi-A_{2}^{\prime \prime}(z) \rho^{2} \frac{1}{8} \sin \varphi+A_{2}^{(4)}(z) \rho^{4} \frac{1}{192} \sin \varphi-A_{2}^{(6)}(z) \rho^{6} \frac{1}{9216} \sin \varphi+\cdots\right\} \\
& +\rho^{3 / 2}\left\{A_{3}(z) \cos \frac{3 \varphi}{2}-A_{3}^{\prime \prime}(z) \rho^{2} \frac{1}{10} \cos \frac{3 \varphi}{2}+A_{3}^{(4)}(z) \rho^{4} \frac{1}{280} \cos \frac{3 \varphi}{2}-A_{3}^{(6)}(z) \rho^{6} \frac{1}{15120} \cos \frac{3 \varphi}{2}+\cdots\right\} \\
& +\rho^{2}\left\{A_{4}(z) \sin 2 \varphi-A_{4}^{\prime \prime}(z) \rho^{2} \frac{1}{12} \sin 2 \varphi+A_{4}^{(4)}(z) \rho^{4} \frac{1}{384} \sin 2 \varphi-A_{4}^{(6)}(z) \rho^{6} \frac{1}{23040} \sin 2 \varphi+\cdots\right\} \\
& +\cdots
\end{aligned}
$$




\subsection{Computation of eigenfunctions and shadows for a crack with homogeneous Neumann BCs}

As in section 2.1, equations (6)-(7) can be solved for $\alpha_{k}=0,1 / 2,1,3 / 2$ with the homogeneous Neumann BCs (4), obtaining $\phi_{1, \ell}, \phi_{2, \ell}, \phi_{3, \ell}, \phi_{4, \ell}$. These yield the following series solution for a straight crack with homogeneous Neumann BCs:

$$
\begin{aligned}
\tau & =\left\{A_{0}(z)-A_{0}^{\prime \prime}(z) \rho^{2} \frac{1}{4}+A_{0}^{(4)}(z) \rho^{4} \frac{1}{64}-A_{0}^{(6)}(z) \rho^{6} \frac{1}{2304}+\cdots\right\} \\
& +\rho^{1 / 2}\left\{A_{1}(z) \sin \frac{\varphi}{2}-A_{1}^{\prime \prime}(z) \rho^{2} \frac{1}{6} \sin \frac{\varphi}{2}+A_{1}^{(4)}(z) \rho^{4} \frac{1}{120} \sin \frac{\varphi}{2}-A_{1}{ }^{(6)}(z) \rho^{6} \frac{1}{5040} \sin \frac{\varphi}{2}+\cdots\right\} \\
& +\rho^{1}\left\{A_{2}(z) \cos \varphi-A_{2}{ }^{\prime \prime}(z) \rho^{2} \frac{1}{8} \cos \varphi+A_{2}{ }^{(4)}(z) \rho^{4} \frac{1}{192} \cos \varphi-A_{2}{ }^{(6)}(z) \rho^{6} \frac{1}{9216} \cos \varphi+\cdots\right\} \\
& +\rho^{3 / 2}\left\{A_{3}(z) \sin \frac{3 \varphi}{2}-A_{3}^{\prime \prime}(z) \rho^{2} \frac{1}{10} \sin \frac{3 \varphi}{2}+A_{3}{ }^{(4)}(z) \rho^{4} \frac{1}{280} \sin \frac{3 \varphi}{2}-A_{3}{ }^{(6)}(z) \rho^{6} \frac{1}{15120} \sin \frac{3 \varphi}{2}+\cdots\right\} \\
& +\cdots
\end{aligned}
$$

Remark 3 Notice that (15) is similar to (14) with the sin functions replaced by the cos functions.

\subsection{The problem with dual shadows associated with integer eigenvalues}

The dual eigenfunctions associated with the negative non-integer eigenvalues (i.e. $\alpha_{k}=-k / 2$ ) and their shadows can be computed by (9)-(10) as shown in (11)-(13). Here, we emphasize the problem of computing dual shadows associated with integer eigenvalues. Consider or example a crack with homogeneous Neumann BCs, for which the first integer dual eigenvalue is $\alpha_{2}=-1$. To compute the first eigenfunction $\psi_{2,0}(\varphi)$, we substitute $\alpha_{2}=-1$ in (9):

$$
(-1)^{2} \psi_{2,0}(\varphi)+\psi_{2,0}^{\prime \prime}(\varphi)=0
$$

The solution of (16) with homogeneous Neumann BCs (4) on the crack faces is:

$$
\psi_{2,0}(\varphi)=\cos (\varphi)
$$

Substitute (17) in (10), with $\alpha_{k}=-1$ and $\ell=2$ to obtain $\psi_{2,2}(\varphi)$ :

$$
\psi_{2,2}(\varphi)+\psi_{2,2}^{\prime \prime}(\varphi)=-\cos (\varphi)
$$

The homogeneous solution of (18) is identical to the particular solution, and there is no solution that satisfies the homogeneous Neumann BCs (4).

Remark 4 This problem appears for all dual shadows associated with integer eigenvalues in the case of a crack with homogeneous Dirichlet or Neumann BCs. It also appears for $V$-notches and for the elasticity system.

\subsection{The multiplicity of the integer dual eigenvalues}

Denote by $\tau^{\left(\alpha_{k}\right)}$ the solution of the Laplace equation associated with the positive eigenvalue $\alpha_{k}$.

$$
\tau^{\left(\alpha_{k}\right)}(\rho, \varphi, z)=\sum_{\ell=0,2,4, \cdots}^{\infty} \partial_{z}^{\ell} A_{k}(z) \rho^{\alpha_{k}+\ell} \phi_{k, \ell}(\varphi)
$$

$\tau^{\left(\alpha_{k=2}=1\right)}$ associated with the second positive eigenvalue $\alpha_{k=2}=1$ is:

$$
\tau^{\left(\alpha_{2}=1\right)}(\rho, \varphi, z)=\rho^{1}\left\{A_{2}(z) \phi_{2,0}(\varphi)+A_{2}^{\prime \prime}(z) \rho^{2} \phi_{2,2}(\varphi)+A_{2}^{(4)}(z) \rho^{4} \phi_{2,4}(\varphi)+\cdots\right\}
$$

The ODEs to determine $\phi_{2, \ell}$ are:

$$
\begin{aligned}
(1)^{2} \phi_{2,0}+\phi_{2,0}^{\prime \prime} & =0 \\
(1+2)^{2} \phi_{2,2}+\phi_{2,2}^{\prime \prime} & =-\phi_{2,0} \\
(1+4)^{2} \phi_{2,4}+\phi_{2,4}^{\prime \prime} & =-\phi_{2,2}
\end{aligned}
$$

Let $K^{\left(\alpha_{j}\right)}$ be the dual solution associated with the negative eigenvalue $-\alpha_{j}$.

$$
K^{\left(\alpha_{j}\right)}(\rho, \varphi, z)=\sum_{\ell=0,2,4, \cdots}^{\infty} \partial_{z}^{\ell} B_{j}(z) \rho^{-\alpha_{j}+\ell} \psi_{j, \ell}(\varphi)
$$


Specifically, $K^{\left(\alpha_{j=2}=-1\right)}$ is:

$$
K^{\left(\alpha_{2}=-1\right)}(\rho, \varphi, z)=\rho^{-1}\left\{B_{2}(z) \psi_{2,0}(\varphi)+B_{2}^{\prime \prime}(z) \rho^{2} \psi_{2,2}(\varphi)+B_{2}^{(4)}(z) \rho^{4} \psi_{2,4}(\varphi)+\cdots\right\}
$$

and the ODEs to determine $\psi_{2, \ell}$ are :

$$
\begin{aligned}
(-1)^{2} \psi_{2,0}+\psi_{2,0}^{\prime \prime} & =0 \\
(-1+2)^{2} \psi_{2,2}+\psi_{2,2}^{\prime \prime} & =-\psi_{2,0} \\
(-1+4)^{2} \psi_{2,4}+\psi_{2,4}^{\prime \prime} & =-\psi_{2,2}
\end{aligned}
$$

As shown in Appendix A in equation (70), $\psi_{2,0}=0$. So (24)-(26) reduce to:

$$
\begin{aligned}
& (-1+2)^{2} \psi_{2,2}+\psi_{2,2}^{\prime \prime}=0 \\
& (-1+4)^{2} \psi_{2,4}+\psi_{2,4}^{\prime \prime}=-\psi_{2,2}
\end{aligned}
$$

and $K^{\left(\alpha_{2}=-1\right)}$ is:

$$
\begin{aligned}
K^{\left(\alpha_{2}=-1\right)}(\rho, \varphi, z) & =\rho^{-1}\left\{0+B_{2}^{\prime \prime}(z) \rho^{2} \psi_{2,2}(\varphi)+B_{2}^{(4)}(z) \rho^{4} \psi_{2,4}(\varphi)+\cdots\right\} \\
& =\rho^{1}\left\{B_{2}^{\prime \prime}(z) \psi_{2,2}(\varphi)+B_{2}^{(4)}(z) \rho^{2} \psi_{2,4}(\varphi)+\cdots\right\}
\end{aligned}
$$

Hence, the primal eigenfunction and shadows that construct $\tau^{\left(\alpha_{2}=1\right)}$ in (20) are identical to the dual shadows that construct $K^{\left(\alpha_{2}=-1\right)}$ in (23). Therefore it is necessary to consider logarithmic terms for the dual solution of the Laplace equation.

\section{Dual shadows with logarithmic terms associated with integer eigenvalues}

For the integer dual eigenvalues we consider logarithmic terms in (22) (see [6]):

$$
K^{\left(\alpha_{j}\right)}(\rho, \varphi, z)=\sum_{\ell=0,2,4,6 \ldots}^{\infty} \partial_{z}^{\ell} B_{j}(z) \rho^{\alpha_{j}+\ell}\left(\psi_{j, \ell}(\varphi)+\log (\rho) \tilde{\psi}_{j, \ell}(\varphi)\right), \quad \alpha_{j} \text { negative integers }
$$

Substituting (29) in (1) one obtains:

$$
\begin{aligned}
\Delta K^{\left(\alpha_{j}\right)}=\rho^{\alpha-2} B(z) & \left\{\log (\rho)\left(\alpha^{2} \tilde{\psi}_{j, 0}(\varphi)+\tilde{\psi}_{j, 0}^{\prime \prime}(\varphi)\right)\right. \\
& \left.+\left(\alpha^{2} \psi_{0}(\varphi)+\psi_{j, 0}^{\prime \prime}(\varphi)+2 \alpha \tilde{\psi}_{j, 0}(\varphi)\right)\right\} \\
+ & \rho^{\alpha} B^{\prime \prime}(z)\left\{\log (\rho)\left((2+\alpha)^{2} \tilde{\psi}_{j, 2}(\varphi)+\tilde{\psi}_{j, 2}^{\prime \prime}(\varphi)+\tilde{\psi}_{j, 0}(\varphi)\right)\right. \\
& \left.\quad+\left((2+\alpha)^{2} \psi_{j, 2}(\varphi)+\psi_{j, 2}^{\prime \prime}(\varphi)+2(2+\alpha) \tilde{\psi}_{j, 2}(\varphi)+\psi_{j, 0}(\varphi)\right)\right\} \\
+ & +\rho^{\alpha+2} B^{(4)}(z)\left\{\log (\rho)\left((4+\alpha)^{2} \tilde{\psi}_{j, 4}(\varphi)+\tilde{\psi}_{j, 4}^{\prime \prime}(\varphi)+\tilde{\psi}_{j, 2}(\varphi)\right)\right. \\
& \left.\quad+\left((4+\alpha)^{2} \psi_{j, 4}(\varphi)+\psi_{j, 4}^{\prime \prime}(\varphi)+2(4+\alpha) \tilde{\psi}_{j, 4}(\varphi)+\psi_{j, 2}(\varphi)\right)\right\} \\
+ & \quad \rho^{\alpha+4} B^{(6)}(z)\left\{\log (\rho)\left((6+\alpha)^{2} \tilde{\psi}_{j, 6}(\varphi)+\tilde{\psi}_{j, 6}^{\prime \prime}(\varphi)+\tilde{\psi}_{j, 4}(\varphi)\right)\right. \\
& \left.\quad+\left((6+\alpha)^{2} \psi_{j, 6}(\varphi)+\psi_{j, 6}^{\prime \prime}(\varphi)+2(6+\alpha) \tilde{\psi}_{j, 6}(\varphi)+\psi_{j, 4}(\varphi)\right)\right\} \\
+\cdots=0 &
\end{aligned}
$$

with homogeneous Dirichlet or Neumann BCs at the crack faces:

$$
\begin{gathered}
\psi_{j, \ell}(\varphi= \pm \pi)=\tilde{\psi}_{j, \ell}(\varphi= \pm \pi)=0, \quad \text { Homogeneous Dirichlet BCs } \\
\psi_{j, \ell}^{\prime}(\varphi= \pm \pi)=\tilde{\psi}_{j, \ell}^{\prime}(\varphi= \pm \pi)=0, \quad \text { Homogeneous Neumann BCs }
\end{gathered}
$$

Equation (30) results in the following set of $1-\mathrm{D}$ recursive ODEs for $\psi_{j, \ell}(\varphi)$ and $\tilde{\psi}_{j, \ell}(\varphi)$ :

$$
\text { For } \ell=0 \text { : }
$$

$$
\begin{aligned}
& \alpha_{j}^{2} \tilde{\psi}_{j, 0}+\tilde{\psi}_{j, 0}^{\prime \prime}=0 \\
& \alpha_{j}^{2} \psi_{j, 0}+\psi_{j, 0}^{\prime \prime}+2 \alpha_{j} \tilde{\psi}_{j, 0}=0
\end{aligned}
$$

For $\ell \geq 2$ :

$$
\begin{aligned}
& \left(\alpha_{j}+\ell\right)^{2} \tilde{\psi}_{j, \ell}+\tilde{\psi}_{j, \ell}^{\prime \prime}+\tilde{\psi}_{j, \ell-2}=0 \\
& \left(\alpha_{j}+\ell\right)^{2} \psi_{j, \ell}+\psi_{j, \ell}^{\prime \prime}+2\left(\alpha_{j}+\ell\right) \tilde{\psi}_{j, \ell}+\psi_{j, \ell-2}=0
\end{aligned}
$$




\subsection{Homogenous Dirichlet BCs}

The first integer dual-eigenvalue is: $\alpha_{2}=-1$, and $K^{\left(\alpha_{2}=-1\right)}$ is:

$$
\begin{aligned}
K^{\left(\alpha_{2}=-1\right)}=\rho^{-1} & \left\{B_{2}(z) \sin (\varphi)-B_{2}^{\prime \prime}(z) \rho^{2} \log (\rho) \frac{\sin (\varphi)}{2}\right. \\
& +B_{2}^{(4)}(z) \rho^{4}\left(-\frac{3 \sin (\varphi)}{64}+\log (\rho) \frac{\sin (\varphi)}{16}\right) \\
& \left.+B_{2}^{(6)}(z) \rho^{6}\left(\frac{7 \sin (\varphi)}{2304}-\log (\rho) \frac{\sin (\varphi)}{384}\right)+\cdots\right\}
\end{aligned}
$$

\subsection{Homogenous Neumann BCs}

For homogeneous Neumann BCs, we have first to consider $\alpha_{0}=0$. For $\alpha_{0}=0$ there is no dual eigenvalue, so we use the logarithmic solution presented in $(29)($ see $[10,6])$ to obtain $K^{\left(\alpha_{0}=0\right)}: K^{\left(\alpha_{0}=0\right)}$ not changed

$$
\begin{aligned}
K^{\left(\alpha_{0}=0\right)}=- & \left\{B_{0}(z) \log \rho+B_{0}^{\prime \prime}(z) \rho^{2} \frac{1}{4}(1-\log \rho)\right. \\
& \left.+B_{0}^{(4)}(z) \rho^{4}\left(-\frac{3}{128}+\log \rho \frac{1}{64}\right)+B_{0}^{(6)}(z) \rho^{6}\left(\frac{11}{13824}-\log \rho \frac{1}{2304}\right)+\cdots\right\}
\end{aligned}
$$

The next integer dual-eigenvalue is $\alpha_{2}=-1$ and for it $K^{\left(\alpha_{2}=-1\right)}$ is:

$$
\begin{aligned}
K^{\left(\alpha_{2}=-1\right)}=\rho^{-1} & \left\{B_{2}(z) \cos (\varphi)-B_{2}^{\prime \prime}(z) \rho^{2} \log (\rho) \frac{\cos (\varphi)}{2}\right. \\
& +B_{2}^{(4)}(z) \rho^{4}\left(-\frac{3 \cos (\varphi)}{64}+\log (\rho) \frac{\cos (\varphi)}{16}\right) \\
& \left.+B_{2}^{(6)}(z) \rho^{6}\left(\frac{7 \sin (\varphi)}{2304}-\log (\rho) \frac{\cos (\varphi)}{384}\right)+\cdots\right\}
\end{aligned}
$$

Remark 5 For non-integer eigenvalues and for positive integer eigenvalues the logarithmic terms in (29) vanish. This is of course in accordance with [2].

\section{Computing EFIFs associated with integer eigenvalues by the quasi-dual function method}

To compute the EFIFs we apply the QDFM [3]. A path integral $\mathcal{Q}_{\rho}\left[\tau, K_{n}^{\left(\alpha_{j}\right)}\right]$ over a cylindrical surface with a radius $\rho$ surrounding the straight singular edge is considered:

$$
\mathcal{Q}_{\rho}\left[\tau, K_{n}^{\left(\alpha_{j}\right)}\right]=\int_{z} \int_{-\pi}^{p i}\left(\partial_{\rho} \tau K_{n}^{\left(\alpha_{j}\right)}-\tau \partial_{\rho} K_{n}^{\left(\alpha_{j}\right)}\right) \rho d \varphi d z
$$

$K_{n}^{\left(\alpha_{j}\right)}$ is composed of the dual eigenfunction and its first $n$ shadow functions.

Since the EFIFs $A_{k}(z)$ are unknown, we approximate it (and also $B_{j}(z)$ ) by a family of orthogonal polynomials in the range $[-1,1]$. For this purpose we use the Jacobi polynomials as follows (see $[12,15])$ :

$$
\begin{aligned}
& A_{k}^{\{p, m\}}(z)=\sum_{i=0}^{p} a_{k_{i}} J^{\{i, m\}}(z) \\
& B_{j}^{\{\ell, m\}}(z)=\frac{1}{2 \pi} \frac{2 \ell+2 m+1}{2^{2 m+1}} \frac{(2 m+\ell) ! \ell !}{((\ell+m) !)^{2}}\left(1-z^{2}\right)^{m} J^{\{\ell, m\}}(z)
\end{aligned}
$$

where $J^{\{\ell, m\}}(z)$ is a Jacobi polynomial of order $m$ and a polynomial degree $\ell$ as follows:

$$
J^{\{\ell, m\}}(z)=(\ell+m) !(\ell+m) ! \sum_{s=0}^{\ell} \frac{1}{s !(\ell+m-s) !(m+s) !(\ell-s) !}\left(\frac{z-1}{2}\right)^{\ell-s}\left(\frac{z+1}{2}\right)^{s}
$$


Remark 6 To avoid the integration over the bases of the cylindrical domain on which we perform the integration, we use $m \geq n$ (see [3]).

Since $n$ is finite, the integral $\mathcal{Q}_{\rho}\left[\tau, K_{n}^{\left(\alpha_{j}\right)}\right]$ is surface-dependent. For the computation of the coefficients $a_{j \ell}$ in (41) we used the $\mathcal{Q}_{\rho_{0}}\left[\tau, K_{n}^{\left(\alpha_{j}\right)}\right]$ integral with a remainder of:

$$
\mathcal{Q}_{\rho}\left[\tau, K_{n}^{\left(\alpha_{j}\right)}\left[B_{j}^{\{\ell, m\}}(z)\right]\right]=a_{j \ell}+\mathcal{O}\left(\rho^{\alpha_{s}-\alpha_{j}+n+2}(1+\log \rho)\right)
$$

where $\alpha_{s}$ is the smallest positive eigenvalue. For a crack with homogeneous Dirichlet BCs the smallest positive eigenvalues is $\alpha_{1}=1 / 2$, and for a crack with homogeneous Neumann BCs the smallest positive eigenvalues is $\alpha_{0}=0$.

Remark 7 For the elasticity problem, the coefficients $a_{k_{i}}$ can be computed by the QDFM with a remainder of $\mathcal{O}\left(\rho^{\alpha_{0}-\alpha_{j}+n+1}(1+\log \rho)\right)$.

\subsection{Analytical example of computation of the integer EFIFs by the QDFM}

In this section we address the extraction of the integer EFIFs for homogeneous Dirichlet and Neumann BCs. We approximate $A_{k}^{\{p, m\}}(z)$ and $B_{j}^{\{\ell, m\}}(z)$ by Jacobi polynomials of order $m=4$ and polynomial degree $p=8$

4.1.1. A crack with homogeneous Dirichlet BCs: To extract $A_{2}(z)$ (associated with $\alpha_{2}=1$ ) for a straight crack with homogeneous Dirichlet BCs, we compute $\mathcal{Q}_{\rho}\left[\tau, K_{n}^{(-1)}\left[B_{2}^{\{\ell, 4\}}(z)\right]\right]$ by (40) with $\tau$ in (14) chosen up to order $\rho^{6}$, for $z \in[-1,1], \ell=0,1$ and different values of $n$ :

$$
\begin{aligned}
& \mathcal{Q}_{\rho}\left[\tau, K_{0}^{(-1)}\left[B_{2}^{\{0,4\}}(z)\right]\right]=a_{2_{0}}-\frac{33}{4} \rho^{2} a_{2_{2}}-\frac{91}{11} \rho^{2} a_{2_{4}}+\cdots=a_{2_{0}}+\mathcal{O}\left(\rho^{2}\right) \\
& \mathcal{Q}_{\rho}\left[\tau, K_{0}^{(-1)}\left[B_{2}^{\{1,4\}}(z)\right]\right]=a_{2_{1}}-\frac{273}{20} \rho^{2} a_{2_{3}}-\frac{189}{13} \rho^{2} a_{2_{5}}+\cdots=a_{2_{1}}+\mathcal{O}\left(\rho^{2}\right) \\
& \mathcal{Q}_{\rho}\left[\tau, K_{2}^{(-1)}\left[B_{2}^{\{0,4\}}(z)\right]\right]=a_{2_{0}}-\frac{1365}{32} \rho^{4} a_{2_{4}}+\frac{1365}{8} \rho^{4} \log \rho a_{2_{4}}+\cdots=a_{2_{0}}+\mathcal{O}\left(\rho^{4}+\rho^{4} \log \rho\right) \\
& \mathcal{Q}_{\rho}\left[\tau, K_{2}^{(-1)}\left[B_{2}^{\{1,4\}}(z)\right]\right]=a_{2_{1}}-\frac{3213}{32} \rho^{4} a_{2_{5}}+\frac{3213}{8} \rho^{4} \log \rho a_{2_{5}}+\cdots=a_{2_{1}}+\mathcal{O}\left(\rho^{4}+\rho^{4} \log \rho\right) \\
& \mathcal{Q}_{\rho}\left[\tau, K_{4}^{(-1)}\left[B_{2}^{\{0,4\}}(z)\right]\right]=a_{2_{0}}+\frac{266475}{128} \rho^{6} a_{2_{6}}-\frac{72675}{32} \rho^{6} \log \rho a_{2_{6}}+\cdots=a_{2_{0}}+\mathcal{O}\left(\rho^{6}+\rho^{6} \log \rho\right) \\
& \mathcal{Q}_{\rho}\left[\tau, K_{4}^{(-1)}\left[B_{2}^{\{1,4\}}(z)\right]\right]=a_{2_{1}}+\frac{820743}{128} \rho^{6} a_{2_{7}}-\frac{223839}{32} \rho^{6} \log \rho a_{2_{7}}+\cdots=a_{2_{1}}+\mathcal{O}\left(\rho^{6}+\rho^{6} \log \rho\right)
\end{aligned}
$$

Following (45)-(50), one notices that the term $\rho^{\alpha_{1}-\alpha_{2}}$ vanishes in the convergence analysis and the convergence rate in the error of the extracted $a_{2 \ell}$ is higher compared to (44).

4.1.2. A crack with homogeneous Neumann BCs: To extract $A_{2}(z)$ (associated with $\alpha_{2}=1$ ) for a straight crack with homogeneous Neumann BCs, we compute $\mathcal{Q}_{\rho}\left[\tau, K_{n}^{(-1)}\left[B_{2}^{\{\ell, 4\}}(z)\right]\right]$ by (40) with $\tau$ in (15) chosen up to order $\rho^{6}$, for $z \in[-1,1], \ell=0,1$ and different values of $n$. Same expressions exactly as (45)-(50) are obtained, i.e. the term $\rho^{\alpha_{1}-\alpha_{2}}$ vanishes in the convergence analysis and the convergence rate in the error of the extracted $a_{2 \ell}$ is higher compared to (44).

\section{Extracting $A_{0}(z)$ :}

To extract $A_{0}(z)$, corresponding to the eigenvalue $\alpha_{0}=0$ we use (38) and compute $\mathcal{Q}_{\rho}\left[\tau, K_{n}^{(0)}\left[B_{2}^{\{\ell, 4\}}(z)\right]\right]$ by (40) with (15) for $z \in[-1,1], \ell=0,1$ and for different values of $n$ :

$$
\begin{aligned}
& \mathcal{Q}_{\rho}\left[\tau, K_{0}^{(0)}\left[B_{0}^{\{0,4\}}(z)\right]\right]=a_{0_{0}}-\frac{33}{4} \rho^{2} a_{0_{2}}+\frac{33}{2} \rho^{2} \log \rho a_{0_{2}}+\cdots=a_{0_{0}}+\mathcal{O}\left(\rho^{2}+\rho^{2} \log \rho\right) \\
& \mathcal{Q}_{\rho}\left[\tau, K_{0}^{(0)}\left[B_{0}^{\{1,4\}}(z)\right]\right]=a_{0_{1}}-\frac{273}{20} \rho^{2} a_{0_{3}}+\frac{273}{10} \rho^{2} \log \rho a_{0_{3}}+\cdots=a_{0_{1}}+\mathcal{O}\left(\rho^{2}+\rho^{2} \log \rho\right) \\
& \mathcal{Q}_{\rho}\left[\tau, K_{2}^{(0)}\left[B_{0}^{\{0.4\}}(z)\right]\right]=a_{0_{0}}+\frac{6825}{32} \rho^{4} a_{0_{4}}-\frac{1365}{8} \rho^{4} \log \rho a_{0_{4}}+\cdots=a_{0_{0}}+\mathcal{O}\left(\rho^{4}+\rho^{4} \log \rho\right) \\
& \mathcal{Q}_{\rho}\left[\tau, K_{2}^{(0)}\left[B_{0}^{\{1,4\}}(z)\right]\right]=a_{0_{1}}+\frac{16065}{32} \rho^{4} a_{0_{5}}-\frac{3213}{8} \rho^{4} \log \rho a_{0_{5}}+\cdots=a_{0_{1}}+\mathcal{O}\left(\rho^{4}+\rho^{4} \log \rho\right) \\
& \mathcal{Q}_{\rho}\left[\tau, K_{4}^{(0)}\left[B_{0}^{\{0.4\}}(z)\right]\right]=a_{0_{0}}-\frac{121125}{64} \rho^{6} a_{0_{6}}+\frac{72675}{64} \rho^{6} \log \rho a_{0_{6}}+\cdots=a_{0_{0}}+\mathcal{O}\left(\rho^{6}+\rho^{6} \log \rho\right) \\
& \mathcal{Q}_{\rho}\left[\tau, K_{4}^{(0)}\left[B_{0}^{\{1,4\}}(z)\right]\right]=a_{0_{1}}-\frac{373065}{64} \rho^{6} a_{0_{7}}+\frac{223839}{64} \rho^{6} \log \rho a_{0_{7}}+\cdots=a_{0_{1}}+\mathcal{O}\left(\rho^{6}+\rho^{6} \log \rho\right)
\end{aligned}
$$

Following (51)-(56), one notices that the convergence rate in the error of the extracted $a_{0_{\ell}}$ is as shown in (44). 


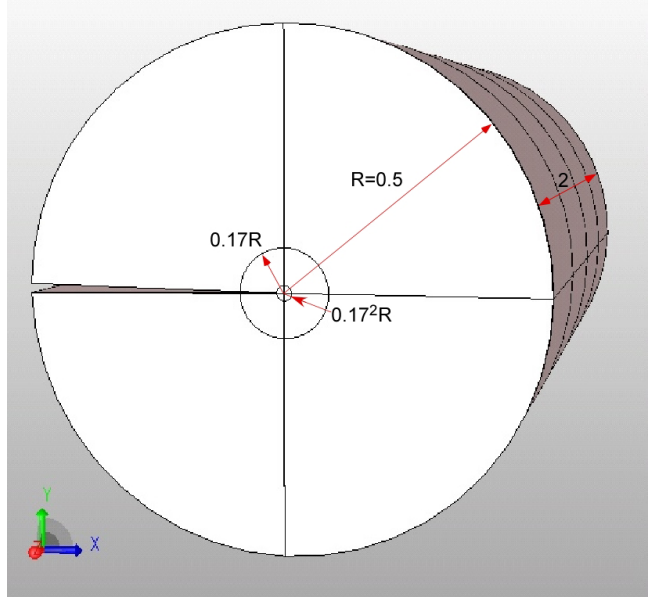

Figure 2. The FE model and the mesh.

\subsection{Extracting $A_{0}$ and $A_{2}$ from $p-F E$ solutions by the $Q D F M$}

Consider a finite elements model of a cracked cylinder of a radius 0.5 and a height $2(z \in[-1,1])$, as shown in Fig 2 .

4.2.1. Homogeneous Dirichlet BCs: On the crack faces of the FE model we prescribe homogeneous Dirichlet BCs. On the cylindrical outer surface the following Dirichlet BC is prescribed:

$$
\tau=\frac{1}{2}\left[\left(1+z+z^{2}+z^{3}+z^{4}\right) \sin \varphi-\frac{1}{8}\left(2+6 z+12 z^{2}\right)\left(\frac{1}{2}\right)^{2} \sin \varphi+\frac{1}{8}\left(\frac{1}{2}\right)^{4} \sin \varphi\right]
$$

On the cylinder's basis $z=-1$ the Dirichlet $B C$ is:

$$
\tau=\rho\left(\sin \varphi-\rho^{2} \sin \varphi+\frac{1}{8} \rho^{4} \sin \varphi\right)
$$

and on the cylinder's basis $z=1$ the Dirichlet $\mathrm{BC}$ is:

$$
\tau=\rho\left(5 \sin \varphi-\frac{5}{2} \rho^{2} \sin \varphi+\frac{1}{8} \rho^{4} \sin \varphi\right)
$$

These boundary conditions result in $A_{2}(z)=1+z+z^{2}+z^{3}+z^{4}$ and $A_{k}=0$ for $k \neq 2$ so that the solution is:

$$
\tau=\rho\left[\left(1+z+z^{2}+z^{3}+z^{4}\right) \sin \varphi-\frac{1}{8}\left(2+6 z+12 z^{2}\right) \rho^{2} \sin \varphi+\frac{1}{8} \rho^{4} \sin \varphi\right]
$$

The integral $\mathcal{Q}_{\rho}\left[\tau^{F E}, K_{n}^{\alpha_{j}}\left[B_{j}^{\{m, \ell\}}(z)\right]\right]$ was computed at $\rho=0.1$ with Jacobi polynomials of order $m=6$ and $\ell=0 \cdots 20$ for different values of $n$. The solution $\tau^{F E}$ was extracted from the p-FE solution with 4387 DOF at $p=8$ having an relative error in energy norm of $0.1 \%$. Figures 3-4 show the extracted $A_{2}^{F E}(z)$ for different values of $n$ and the relative error in the extracted $A_{2}^{F E}(z)$ in percentage. As expected, the error in the extracted EFIFs decreases as the number of the shadow functions $n$ that construct the quasi dual function $K$ increases.

4.2.2. Homogeneous Neumann BCs: Consider again the FE model shown in in Fig 2. This time we prescribe on the domain's surfaces Dirichlet boundary conditions associated with an exact solution having $A_{0}(z)=1+z+z^{2}+z^{3}+z^{4}$, $A_{2}(z)=$ $-2\left(1+z+z^{2}+z^{3}+z^{4}\right)$ and $A_{k}=0$ for $k \neq 0,2$ in (15). Homogeneous Neumann BCs are prescribed on the crack faces. These boundary conditions result in an exact solution given by:

$$
\begin{aligned}
\tau= & \left\{\left(1+z+z^{2}+z^{3}+z^{4}\right)-\frac{1}{4} \rho^{2}\left(2+6 z+12 z^{2}\right)+\frac{3}{8} \rho^{4}\right\} \\
+ & +\rho^{1}\left\{-2\left(1+z+z^{2}+z^{3}+z^{4}\right) \cos \varphi+\frac{1}{2} \rho^{2}\left(1+3 z+6 z^{2}\right) \cos \varphi-\frac{1}{4} \rho^{4} \cos \varphi\right\}
\end{aligned}
$$

The integral $\mathcal{Q}_{\rho}\left[\tau^{F E}, K_{n}^{\alpha_{j}}\left[B_{j}^{\{m, \ell\}}(z)\right]\right]$ was computed at $\rho=0.1$ with Jacobi polynomials of order $m=6$ and $\ell=0 \cdots 20$ for different values of $n$. $\tau^{F E}$ was extracted from the p-FE solution with 5372 DOF at $p=8$ having a relative error in energy 


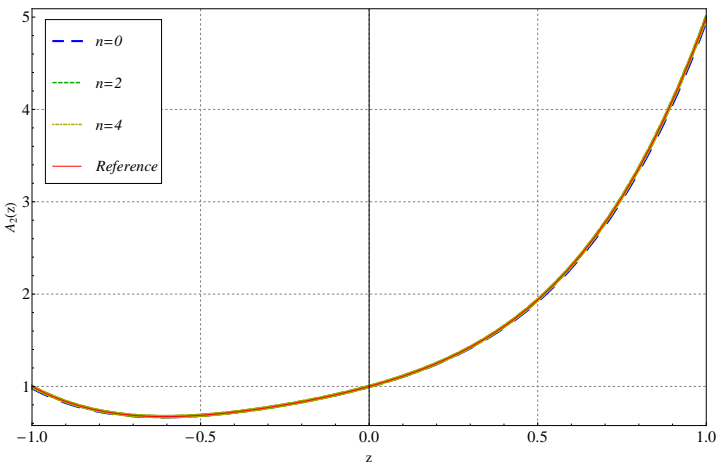

Figure 3. $A_{2}(z)$ for different values of $n$ and the exact function - homogeneous Dirichlet BCs.

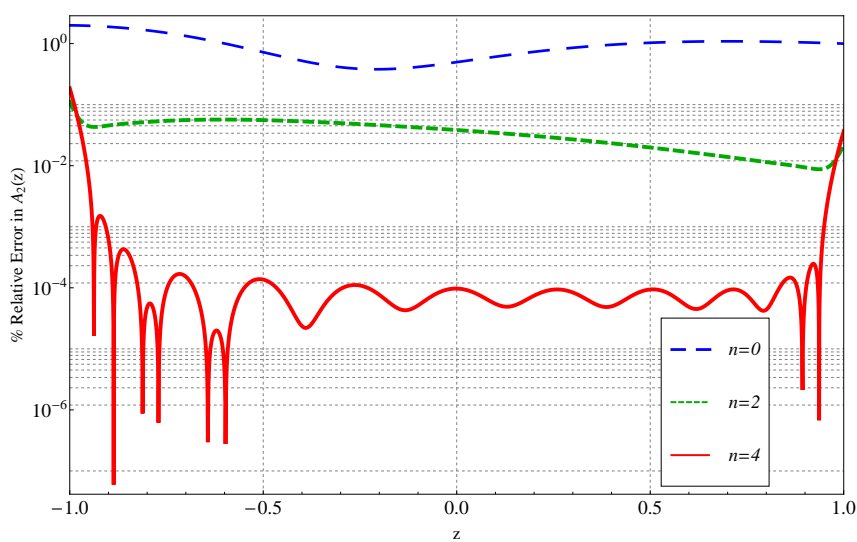

Figure 4. Relative error in the extracted $A_{2}(z)$ in percentage - homogeneous Dirichlet BCs.

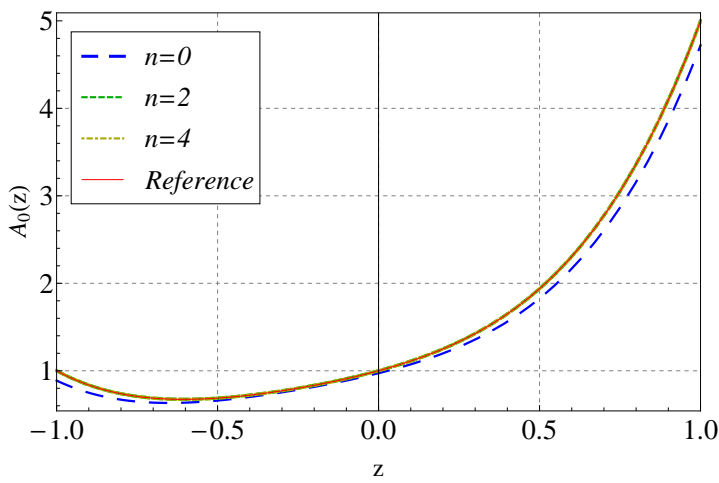

Figure 5. $A_{0}(z)$ obtained for different values of $n$ and the exact function - homogeneous Neumann BCs.

norm of $0.07 \%$. Figures $5-8$ show the extracted $A_{0}^{F E}(z)$ and $A_{2}^{F E}(z)$ for different values of $n$ and the relative error in the extracted $A_{0}^{F E}(z)$ and $A_{2}^{F E}(z)$ in percentage. As expected, the error in the extracted EFIFs decreases as the number of the shadow functions $n$ that construct the quasi dual function $K$ increases.

\section{Summary and conclusions}

We presented the dual solution of the Laplace equation in the vicinity of straight crack. It was demonstrated that for the dual integer eigenfunctions and their shadow functions it contains logarithmic terms. We extended the QDFM for extracting the EFIFs in the vicinity of a straight singular edge for the EFIFs that depend on the integer eigenvalues. The formulation and performance of the method is demonstrated on the simplified Laplace equation. Although the QDFM is a mildly-surface-dependent integral, 


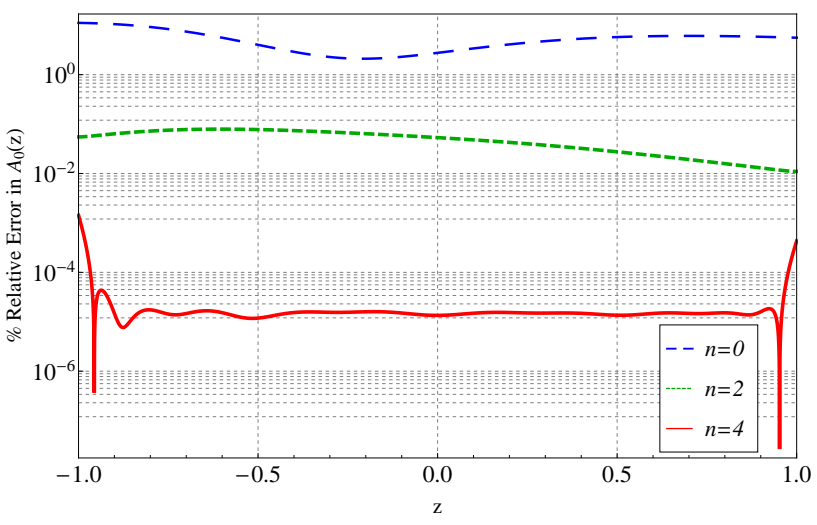

Figure 6. Relative error in the extracted $A_{0}(z)$ in percentage - homogeneous Neumann BCs.

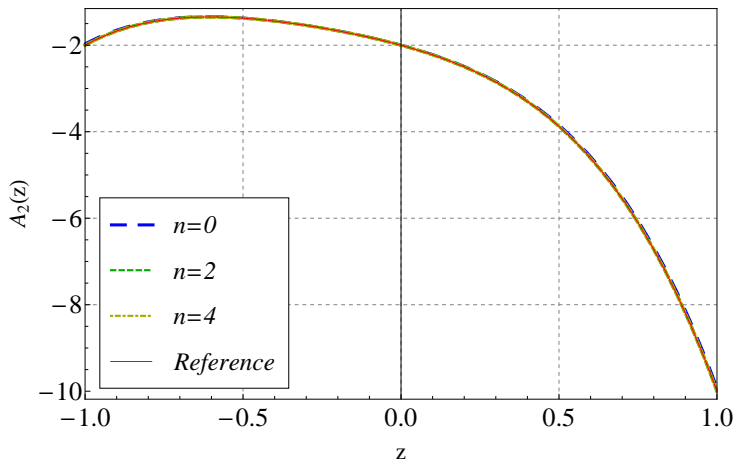

Figure 7. $A_{2}(z)$ for different values of $n$ and the exact function - homogeneous Neumann BCs.

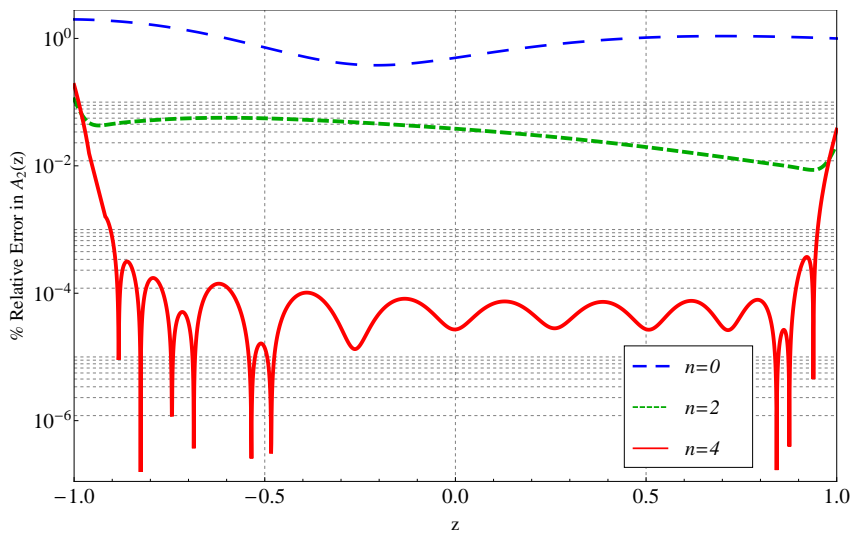

Figure 8. Relative error in the extracted $A_{2}(z)$ in percentage - homogeneous Neumann BCs.

we demonstrated that by using a proper quasi-dual function $K_{n}^{\left(\alpha_{j}\right)}$ and proper extraction functions $B_{j}(z)$, one may extract the functional representation of the EFIFs $A_{k}(z)$ accurately and efficiently.

Because, in general, only a FE approximation of the solution is available,the QDFM in conjunction with $p$-FE methods provide highly accurate EFIFs. These were obtained as a function along the straight edge, and are much more accurate compared to the FE solution (superconvergence property). Another major advantage is the possibility to use the FE solution on a cylindrical surface at a distance from the singular edge, thus it is not necessary to have a refined FE mesh in the vicinity of the singularity (which is a complicated and tedious task in 3-D domains).

The presented methods may be extended to the elasticity system and to circular singular edges following the same methodology presented herein. 


\section{Acknowledgement}

The first and third authors gratefully acknowledge the support of this work by the Israel Science Foundation (grant No. 444/10).

\section{A. Computation of the dual functions for the crack problem - Multiplicity of the Integer Dual eigenvalues}

The set of "dual eigenvalues" for the crack problem is given by the sequence $\left(\alpha_{k}\right)_{k \in \mathbb{N}^{*}}$ with $\alpha_{k}=-\frac{k}{2}$. We introduce the coefficients

$$
\lambda_{k, \ell}=\alpha_{k}+l \text {, }
$$

where $\lambda_{k, \ell} \in \frac{1}{2} \mathbb{Z}=\left\{\frac{p}{2} \mid p \in \mathbb{Z}\right\}$. In the following subsections, we solve the Sturm-Liouville equation

$$
\Phi_{k, \ell}^{\prime \prime}+\lambda_{k, \ell}^{2} \Phi_{k, \ell}=f \quad \text { in }(-\pi, \pi)
$$

with either homogeneous Dirichlet or Neumann boundary conditions. In both cases, we adopt a shooting method to reduce the Sturm-Liouville problem to an initial value problem. We refer also to [9, §3.2.2] for such an approach where the author computes singularities associated with a Neumann problem for the Laplacian set in a sector.

\section{A.1. The Sturm-Liouville problem with Dirichlet boundary conditions}

We write the problem (??)-(3) when $\ell \in 2 \mathbb{N}^{*}$ as

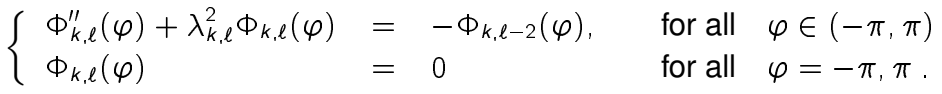

When $\lambda_{k, \ell}=0$, the Sturm-Liouville problem (62) is well-posed. However, when $\lambda_{k, \ell} \in \frac{1}{2} \mathbb{Z}^{*}$, the ODE (62) has a solution provided the right-hand side $\Phi_{k, \ell-2}$ satisfies a compatibility condition (64).

Proof 1 Let $k \in \mathbb{N}^{*}$ and $\ell \in 2 \mathbb{N}^{*}$. We set $\Phi_{k, 0}(\varphi)=c_{k} \sin \left(\alpha_{k} \varphi\right)$, where $c_{k}$ is an arbitrary constant.

(i) Assume that $\lambda_{k, \ell}=0$. Then, the ODE (62) admits the unique solution

$$
\Phi_{k, \ell}(\varphi)=-\int_{-\pi}^{\varphi}(\varphi-s) \Phi_{k, \ell-2}(s) \mathrm{d} s+\frac{(\varphi+\pi)}{2 \pi} \int_{-\pi}^{\pi}(\pi-s) \Phi_{k, \ell-2}(s) \mathrm{d} s .
$$

(ii) Assume that $\lambda_{k, \ell} \in \frac{1}{2} \mathbb{Z}^{*}$, then the ODE (62) admits solutions iff the data $\Phi_{k, \ell-2}$ satisfies the compatibility condition

$$
\int_{-\pi}^{\pi} \sin \left(\lambda_{k, \ell} s\right) \Phi_{k, \ell-2}(s) d s=0
$$

Under the condition (64), the ODE (62) admits the following solutions

$$
\Phi_{k, \ell}(\varphi)=c \sin \left(\lambda_{k, \ell} \varphi\right)-\frac{1}{\lambda_{k, \ell}} \int_{-\pi}^{\varphi} \sin \left(\lambda_{k, \ell}(\varphi-s)\right) \Phi_{k, \ell-2}(s) \mathrm{d} s
$$

where $c$ is an arbitrary constant.

Proof 2 (i) When $\lambda_{k, \ell}=0$, the ODE (62) has a unique solution $\Phi_{k, \ell}$ and straightforward computations lead to (63). (ii) We fix $k, \ell$ and we set $\lambda=\lambda_{k, \ell}$ and $f=-\Phi_{k, \ell-2}$. We first reduce the ODE (62) to an initial value problem : we consider the Sturm-Liouville problem with an auxiliary boundary condition

$$
\begin{cases}\Phi_{\beta}^{\prime \prime}(\varphi)+\lambda^{2} \Phi_{\beta}(\varphi) & =f(\varphi), \quad \text { for all } \varphi \in(-\pi, \pi) \\ \Phi_{\beta}(-\pi) & =0 . \\ \Phi_{\beta}^{\prime}(-\pi) & =\beta .\end{cases}
$$

Here $\beta$ is a given complex parameter. We set $\Psi_{\beta}=\left(\Phi_{\beta}, \Phi_{\beta}^{\prime}\right)^{T}$. Then, the function $\Psi_{\beta}$ satisfies the ODE of order 1

$$
\begin{cases}\Psi_{\beta}^{\prime}(\varphi)-A \Psi_{\beta}(\varphi) & =F(\varphi), \quad \text { for all } \varphi \in(-\pi, \pi) \\ \Psi_{\beta}(-\pi) & =\Omega_{\beta}\end{cases}
$$

Here,

$$
A=\left(\begin{array}{cc}
0 & 1 \\
-\lambda^{2} & 0
\end{array}\right), \quad F=(0, f)^{T}, \quad \Omega_{\beta}=(0, \beta)^{T}
$$


For all given $\beta$, the ODE (67) admits the unique solution $\Psi_{\beta}$ :

$$
\Psi_{\beta}(\varphi)=e^{(\varphi+\pi) A} \Omega_{\beta}+\int_{-\pi}^{\varphi} e^{(\varphi-s) A} F(s) \mathrm{d} s
$$

Here, the resolvent $e^{(\varphi-s) A}$ writes

$$
e^{(\varphi-s) A}=\left(\begin{array}{cc}
\cos \lambda(\varphi-s) & \frac{1}{\lambda} \sin \lambda(\varphi-s) \\
-\lambda \sin \lambda(\varphi-s) & \cos \lambda(\varphi-s)
\end{array}\right)
$$

Hence, there holds

$$
\Phi_{\beta}(\varphi)=\beta \frac{1}{\lambda} \sin \lambda(\varphi+\pi)+\frac{1}{\lambda} \int_{-\pi}^{\varphi} \sin (\lambda(\varphi-s)) f(s) \mathrm{d} s .
$$

The problem (62) has a unique solution iff there exists a unique $\beta$ such that :

$$
\Phi_{\beta}(\pi)=0,
$$

i.e.

$$
\beta \sin 2 \lambda \pi=-\int_{-\pi}^{\pi} \sin (\lambda(\pi-s)) f(s) \mathrm{d} s
$$

Hence, when $\lambda \in \frac{1}{2} \mathbb{Z}^{*}$, there holds $\sin 2 \lambda \pi=0$ and the equation (69) admits a solution iff the compatibility condition (64) is satisfied. Then, the solutions of the ODE (62) are given by a sum of a particular solution and any solution of the homogeneous equation, (65).

\section{A.2. Application to the computation of the dual functions}

A.2.1. Computations of the functions $\Phi_{2, l}, I=0,2,4$ We set $k=2$. There holds $\alpha_{2}=-1$ and we $\operatorname{set} \Phi_{2,0}(\varphi)=-c_{2} \sin \varphi$.

We set $\ell=2$. There holds $\lambda_{2,2}=1$. Then, the compatibility condition (64) writes $\pi c_{2}=0$ and is satisfied iff $c_{2}=0$. We set $c_{2}=0$, hence

$$
\Phi_{2,0}=0
$$

and the compatibility condition (64) is satisfied when $(k, \ell)=(2,2)$. Then the ODE (62) admits the following solutions

$$
\Phi_{2,2}(\varphi)=c \sin \varphi
$$

where $c$ is an arbitrary constant.

We set $\ell=4$. Then, the compatibility condition (64) is satisfied since

$$
\int_{-\pi}^{\pi} \sin 3 s \sin s d s=0
$$

Hence, the ODE (62) admits the following solutions

$$
\Phi_{2,4}(\varphi)=d \sin (3 \varphi)-\frac{c}{3} \int_{-\pi}^{\varphi} \sin (3(\varphi-s)) \sin s d s
$$

where $d$ is an arbitrary constant, i.e.

$$
\Phi_{2,4}(\varphi)=\left(d+\frac{c}{24}\right) \sin (3 \varphi)-\frac{c}{8} \sin \varphi
$$

\section{A.3. The Sturm-Liouville problem with Neumann boundary conditions}

Here, we consider the Sturm-Liouville problem with homogeneous Neumann boundary conditions

$$
\left\{\begin{array}{lll}
\Phi_{k, \ell}^{\prime \prime}(\varphi)+\lambda_{k, \ell}^{2} \Phi_{k, \ell}(\varphi) & =f(\varphi), & \text { for all } \varphi \in(-\pi, \pi) \\
\Phi_{k, \ell}^{\prime}(\varphi) & =0 & \text { for all } \varphi=-\pi, \pi .
\end{array}\right.
$$

When $\lambda_{k, \ell} \in \frac{1}{2} \mathbb{Z}^{*}$ (resp. $\lambda_{k, \ell}=0$ ), the Sturm-Liouville problem (71) has a solution provided the right-hand side $f$ satisfies a compatibility condition (74) (resp. (72)).

Proof 3 Let $f \in \mathcal{C}([-\pi, \pi])$. Let $k \in \mathbb{N}^{*}$ and $\ell \in 2 \mathbb{N}^{*}$.

(i) Assume that $\lambda_{k, \ell}=0$. Then, the ODE (71) admits a solution iff the data $f$ satisfies the compatibility condition

$$
\int_{-\pi}^{\pi} f(s) \mathrm{d} s=0
$$


Under the condition (72), the ODE (71) admits the following solutions

$$
\Phi_{k, \ell}(\varphi)=c+\int_{-\pi}^{\varphi}(\varphi-s) f(s) \mathrm{d} s
$$

where $c$ is an arbitrary constant.

(ii) Assume that $\lambda_{k, \ell} \in \frac{1}{2} \mathbb{Z}^{*}$, then the ODE (71) admits solutions iff the data $f$ satisfies the compatibility condition

$$
\int_{-\pi}^{\pi} \cos (\lambda(\pi-s)) f(s) \mathrm{d} s=0
$$

Under the condition (74), the ODE (71) admits the following solutions

$$
\Phi_{k, \ell}(\varphi)=c \cos \left(\lambda_{k, \ell} \varphi\right)+\frac{1}{\lambda_{k, \ell}} \int_{-\pi}^{\varphi} \sin \left(\lambda_{k, \ell}(\varphi-s)\right) f(s) \mathrm{d} s
$$

where $c$ is an arbitrary constant.

Proof 4 (i) When $\lambda_{k, \ell}=0$, the ODE (71) admits a solution if and only if $f$ satisfies the compatibility condition (72). Then straightforward computations lead to (73).

(ii) We fix $k, \ell$ and we set $\lambda=\lambda_{k, \ell}$. We consider the Sturm-Liouville problem with an auxiliary boundary condition

$$
\begin{cases}\Phi_{\beta}^{\prime \prime}(\varphi)+\lambda^{2} \Phi_{\beta}(\varphi) & =f(\varphi), \quad \text { for all } \varphi \in(-\pi, \pi) \\ \Phi_{\beta}(-\pi) & =\beta, \\ \Phi_{\beta}^{\prime}(-\pi) & =0 .\end{cases}
$$

Here $\beta$ is a given complex parameter. We set $\Psi_{\beta}=\left(\Phi_{\beta}, \Phi_{\beta}^{\prime}\right)^{T}$. Then, the function $\Psi_{\beta}$ satisfies the ODE of order 1

$$
\begin{cases}\Psi_{\beta}^{\prime}(\varphi)-A \Psi_{\beta}(\varphi) & =F(\varphi), \text { for all } \varphi \in(-\pi, \pi) \\ \Psi_{\beta}(-\pi) & =\Upsilon_{\beta}\end{cases}
$$

Here,

$$
A=\left(\begin{array}{cc}
0 & 1 \\
-\lambda^{2} & 0
\end{array}\right), \quad F=(0, f)^{T}, \quad \Upsilon_{\beta}=(\beta, 0)^{T} .
$$

For all given parameter $\beta$, the ODE (77) admits the unique solution $\Psi_{\beta}$ (68) :

$$
\Psi_{\beta}(\varphi)=e^{(\varphi+\pi) A} \Upsilon_{\beta}+\int_{-\pi}^{\varphi} e^{(\varphi-s) A} F(s) \mathrm{d} s
$$

Hence, there holds

$$
\Phi_{\beta}(\varphi)=\beta \cos \lambda(\varphi+\pi)+\frac{1}{\lambda} \int_{-\pi}^{\varphi} \sin (\lambda(\varphi-s)) f(s) \mathrm{d} s .
$$

The problem (71) has a unique solution if there exists a unique $\beta$ such that :

$$
\Phi_{\beta}^{\prime}(\pi)=0 \text {, }
$$

i.e.

$$
\beta \lambda \sin 2 \lambda \pi=\int_{-\pi}^{\pi} \cos (\lambda(\pi-s)) f(s) \mathrm{d} s .
$$

Hence, when $\lambda \in \frac{1}{2} \mathbb{Z}^{*}, \sin 2 \lambda \pi=0$ and the equation (79) admits a solution $\beta$ if the compatibility condition (74) is satisfied. Then, the solutions of the ODE (71) are given by a sum of a particular solution and any solution of the homogeneous equation, (75).

\section{References}

1. E. Christodoulou, C. Elliotis, C. Xenophontos, and G.C. Georgiou. The singular function boundary integral method for 3-d Laplacian problems with a boundary straight edge singularity. Appl. Math. and Comp., 219:1073-1081, 2012.

2. M. Costabel, M. Dauge, and R. Duduchava. Asymptotics without logarithmic terms for crack problems. Communication in PDEs, 28(56):869-926, 2003.

3. M. Costabel, M. Dauge, and Z. Yosibash. A quasidual function method for extracting edge stress intensity functions. SIAM Jour. Math. Anal., 35(5):1177-1202, 2004.

4. B. Cotterell and JR. Rice. On a slightly curved or kinked crack. Int. Jour. Fracture, 16:155-169, 1980. 
5. M. Dauge. Elliptic boundary value problems in corner domains - smoothness and asymptotics of solutions. Lecture notes in Mathematics 1341, Springer-Verlag, Heidelberg, 1988.

6. M. Dauge, P. Dular, L. Krähenbühl, V. Péron, R. Perrussel, and C. Poignard. Corner asymptotics of the magnetic potential in the eddycurrent model. Math. Meth. Appl. Sci., 2013. published online.

7. R.J. Hartranft and G.C. Sih. The use of eigenfunction expansions in the general solution of three-dimensional crack problems. Jour. Math. Mech., 19(2):123-138, 1967.

8. D. Leguillon and S. Murer. Crack deflection in a biaxial stress state. Int. Jour. Fracture, 150:75-90, 2008.

9. A. Makhlouf. Justification et amélioration de modèles d'antennes patch par la méthode des développements asymptotiques raccordés. $\mathrm{PhD}$ thesis, Institut de Mathématiques de Toulouse, France, 2008.

10. V.G. Maz'ya and B.A. Plamennevskii. On the coe?cients in the asymptotic of solutions of the elliptic boundary problem in domains with conical points. Amer. Math. Soc. Trans., 123:57-88, 1984.

11. B. Nkemzi and M. Jung. Flux intensity functions for the Laplacian at polyhedral edges. Int. Jour. Fracture, 175:167-185, 2012.

12. N. Omer, Z. Yosibash, M. Costabel, and M. Dauge. Edge flux intensity functions in polyhedral domains and their extraction by a quasidual function method. Int. Jour. Fracture, 129:97-130, 2004.

13. T. von Petersdorff and E. P. Stephan. Decompositions in edge and corner singularities for the solution of the Dirichlet problem of the Laplacian in a polyhedron. Math. Nachr., 149:71-103, 1990.

14. M. L. Williams. Stress singularities resulting from various boundary conditions in angular corners of plates in extension. Trans. ASME, Jour. Appl. Mech., 19:526-528, 1952.

15. Z. Yosibash, N. Omer, M. Costabel, and M. Dauge. Edge stress intensity functions in polyhedral domains and their extraction by a quasidual function method. Int. Jour. Fracture, 136:37 - 73, 2005. 